\title{
THE EFFECTIVE OF DIFFERENT EXCITATION WAVELENGTHS ON THE IDENTIFICATION OF PLANT SPECIES BASED ON FLUORESCENCE LIDAR
}

\author{
Jian Yang ${ }^{\text {a*}}$, Wei Gong a,b, Shuo Shi ${ }^{\text {a,b,c }}$, Lin Du ${ }^{\text {a,d }}$, Jia Sun ${ }^{\text {a, }}$, Shalei Songe \\ ${ }^{\text {a }}$ Key Laboratory of Information Engineering in Surveying, Mapping, and Remote Sensing, Wuhan University, Wuhan, China - \\ wind_yang@whu.edu.cn; weigong@whu.edu.cn; shishuo@whu.edu.cn; linyufocus@ foxmail.com; helena@whu.edu.cn

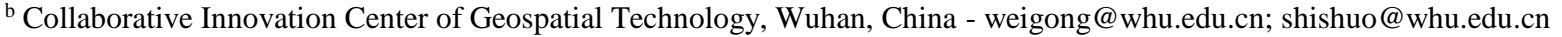 \\ ${ }^{\mathrm{c}}$ School of Resource and Environmental Sciences, Wuhan University, Wuhan, China - shishuo@ whu.edu.cn \\ ${ }^{d}$ School of Physics and Technology, Wuhan University, Wuhan, China - linyufocus@ foxmail.com \\ e Wuhan Institute of Physics and Mathematics, Chinese Academy of Sciences, Wuhan, China - songshalei@gmail.com
}

\section{Commission I, WG I/2}

KEY WORDS: Laser-induced fluorescence, Excitation wavelength, Multivariate analysis, Remote sensing, classification

\begin{abstract}
:
Laser-induced fluorescence (LIF) served as an active technology has been widely used in many field, and it is closely related to excitation wavelength (EW). The objective of this investigation is to discuss the performance of different EWs of LIF LiDAR in identifying plant species. In this study, the 355, 460 and $556 \mathrm{~nm}$ lasers were utilized to excite the leaf fluorescence and the fluorescence spectra were measured by using the LIF LiDAR system built in the laboratory. Subsequently, the principal component analysis (PCA) with the help of support vector machine (SVM) was utilized to analyse fluorescence spectra. For the three EWs, the overall identification rates of the six plant species were $80 \%, 83.3 \%$ and $90 \%$. Experimental results demonstrated that $556 \mathrm{~nm}$ excitation light source is superior to 355 and $460 \mathrm{~nm}$ for the classification of the plant species for the same genus in this study. Thus, an appropriate excitation wavelength should be considered when the LIF LiDAR was utilized in the field of remote sensing based on the LIF technology.
\end{abstract}

\section{INTRODUCTION}

The diversity of vegetation is very important to the entire ecological system. In recent decades, a large number of technologies (Gong, et al. 2012; Vauhkonen et al. 2013), including passive and active remote sensing, have been proposed to monitor vegetation species. For passive remote sensing, spectral reflectance measurements of vegetation territory can provide the relative area covered by vegetation chlorophyll with respect to the total area being scanned (Du et al. 2014). These data are valuable, particularly when monitoring changes in the local vegetation cover. However, the using of the acquired spectral data disenables the accurate classification and identification of plant species or groups. In addition, this technology was also restricted by many other factors, such as weather condition, measurement time etc. (Wu et al. 2009). Hence, researchers hope to be able to develop new techniques that can overcome these deficiencies. Then, LiDAR was proposed in the field of remote sensing (Koukoulas et al. 2005). It was not limited by the weather condition and measurement time, and displayed the advantage of high temporal-spatial resolution and non-destructive. Therefore, it has been widely applied in vegetation monitoring. In addition, Gong et al. (2012) proposed multi-wavelength canopy LiDAR (MWCL) containing both the spatial and spectral information for remote sensing of vegetation. This technology has been successfully utilized to distinguish the coniferous from broad-leaf forest and can be employed to monitor the nutrient stress of crops.

In the past decade years, an active remote sensing technology was proposed by Chappelle et al. (1984), which utilizes ultraviolet (UV) light as the excitation light source to acquire plant fluorescent characteristics. As part of active remote sensing technology, the feasibility of laser-induced fluorescence (LIF) for detecting the status of vegetation has been tested in aircrafts (Rogers et al. 2012). LIF technology exhibits the advantages of quick response, high sensitivity, and nondestructive property (Apostol et al. 2007). Then, LIF has the potential to become a significant approach for vegetation detection (Günther et al. 1994). At present, LIF is usually employed with an emission spectra measured at UV excitation wavelength (EW) because the fluorescence quantum efficiency of the fluorophore at $355 \mathrm{~nm}$ is higher than those at other EWs (Agati 1998). A lot of researches have taken place since the early studies of Chappelle et al., where LIF was mainly used to monitor the status and biomass of vegetation with UV excitation (Ramos and Lagorio 2004). Chappelle et al. (1985) analysed the differences among monocots, dicots and hardwoods based on the characteristics of vegetation fluorescence peaks. The LIF is that the energy of a specific wavelength was absorbed by fluorophore and emitted the light at longer wavelengths. Thus, the LIF technology is closely related to EW. At present, a large number of investigations have been conducted on the correlation between fluorescence spectra and different EWs (Yang et al. 2016). To data, however, few studies have been done on the EWs analysis of LIF LiDAR for the classification of plant species based on the principal component analysis (PCA) combined with support vector machine (SVM).

Therefore, this study is mainly to investigate the effect of EW on the identifying of plant species based on LIF technology. The LIF LiDAR was built in laboratory, and three different excitation light sources (355, 450 and $556 \mathrm{~nm}$ lasers) were utilized to induce the leaf fluorescence of plant species. These fluorescence spectra data measured were stored in a

${ }^{*}$ Corresponding author 
fluorescence spectral database. PCA was utilized to analyse these fluorescence spectra by extracting the major attributes and reducing the dimensionality of variables. It found that over $90 \%$ of the total variance can be explained by using the first three principal components (PCs). Then, SVM algorithm based on these PCs served as input variables was employed to identify these plant species included those of the same family.

\section{MATERIALS AND METHODS}

\subsection{Samples}

In this experiment, the leaves of eight typical plant species (including Cerasus yedoensis, Cerasus dielsiana, Cinnamomum kotoense, Salix babylonica Linn., llex chinensis Sims, Magnolia denudata Desr.) were gathered from the subtropical zone of central China at $30^{\circ} 32^{\prime} \mathrm{N}$ and $114^{\circ} 21^{\prime} \mathrm{E}$. The data of sample is 11 July 2015 . These samples were sealed in plastic bags, kept in an ice chest, and then immediately transported to the laboratory for LIF spectra measurements.

\subsection{Experimental system}

The schematic of the experimental instrument is shown in Figure 1. The instrument consists of three main parts: the signal acquisition part, the optical receiver assembly, and the excitation light source. The UV excitation light source is 355 $\mathrm{nm}$ and is emitted by a frequency-tripled Nd:YAG laser. The 460 and $556 \mathrm{~nm}$ lasers were manufactured by Spectra-Physics. The three EWs are relatively easy to be obtained, and represent the UV, blue, and green excitation light. Maksutov-Cassegrain telescope was used to collect the emission fluorescence signals of leaf. Then, a single-mode optical fiber with a diameter of 200 um was utilized to transmit the fluorescence collected between the telescope and spectrograph. The slit of the spectrograph was set to $0.5 \mathrm{~mm}$. LIF signal variation with wavelength was detected by using an intensified charge-coupled device (ICCD) camera. The fluorescence data was stored in a personal computer. In this study, the spectral range of fluorescence, which was excited by $355 \mathrm{~nm}$ laser, was $360-800 \mathrm{~nm}$ and with a $0.5 \mathrm{~nm}$ sampling interval. The fluorescence excited by 460 and $556 \mathrm{~nm}$ ranged from 630 to $790 \mathrm{~nm}$ and sampling interval was $0.5 \mathrm{~nm}$ (Figure 2).

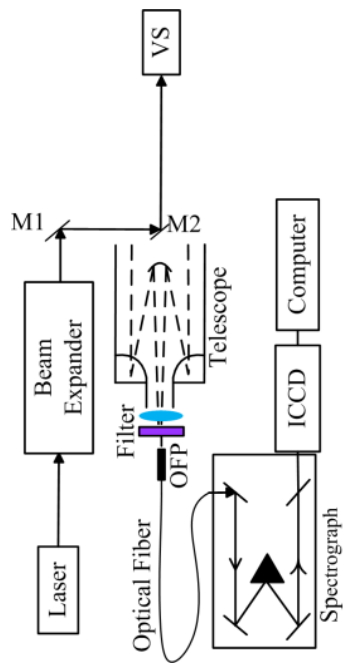

Figure 1. Schematic of LIF Lidar. $M 1$ and $M 2$ : completely reflecting mirror, $V S$ : vegetation sample, $O F B$ : optical fiber probe

\subsection{Data analysis and methods}

The fluorescence spectra of leaves were measured by utilizing the LIF instrument (Figure 1), and the data collected were stored in a personal computer. To reduce the systematic error and the effects of laser energy fluctuation, all fluorescence spectra were repeatedly measured nine times and calculated the average. In this study, of which 240 sets of fluorescence spectra data were acquired from each of the three EWs (355, 460 and $556 \mathrm{~nm})$. To reduce the variables of the fluorescence spectra datasets, PCA, a traditional method of multivariate analysis, was utilized to analyse fluorescence spectra (Agarwal et al. 2003)

The PCA is a powerful multivariate statistical data analysis technique. The multiple variables can be converted to a few variables, in which each principal component (PC) is a linear combination of the primitive variables. These PCs extracted the most important information containing in the variables. Thus, this method has been widely used in many research fields. The detail introduce can be found in reference ( $\mathrm{Yi}$ et al. 2007). Therefore, PCA was employed to extract the feature vectors of fluorescence spectra by analysing major attributes.

SVM was then utilized to analyse the performance of fluorescence spectra excited by different EWs for the classification of plant species. The SVM is a classical supervised learning algorithm with a strong theoretical foundation in statistical theory which can convert low dimensional characteristics to high dimensional characteristics for recognizing some complex targets. The detailed describing can be referenced. The SVM exhibits the special advantages in heterogeneous classes for small samples and high dimensional cases. The kernel function is a critical factor for SVM analysis, and then the radial basis function (RBF) was utilized in this study. It can be presented follows:

$$
K\left(x_{i}, x_{j}\right)=\exp \left(-\gamma\left\|x_{j}-x_{i}\right\|^{2}\right)
$$

Where $\gamma=$ kernel parameter,

$$
\begin{aligned}
& x_{j}=\text { test data } \\
& x_{i}=\text { training inputs }
\end{aligned}
$$

All new feature variables were then randomly separated into two parts: $75 \%$ as the training set to train the SVM model and $25 \%$ as the validation set to test the model tentatively. Before analysis, these fluorescence spectra were denoised and smoothened by using a moving-window polynomial fitting and wavelet transform, respectively.

\section{RESULTS AND DISCUSSION}

The 355, 460 and $556 \mathrm{~nm}$ excitation light sources were utilized to excite the leaf fluorescence spectra of different plant species. The average normalized fluorescence spectra of all samples of the same species, which was excited by different EWs, are shown in Figure 2. Figure 2 demonstrates that the leaf fluorescence spectra of different plant species were similar at single-excitation wavelength $(355,460$ or $556 \mathrm{~nm})$. All of the fluorescence spectra display two main fluorescence peaks bands at $680 \sim 690 \mathrm{~nm}$ and $730 \sim 740 \mathrm{~nm}$. The centre wavelengths of the two fluorescence peaks are 685 and $740 \mathrm{~nm}$, respectively. As concluded by Chappelle et al. (1984, 1985), two fluorescence peaks at 685 and $740 \mathrm{~nm}$ are attributed to the centre pigment of Photosystem II (chlorophyll a) and antennae chlorophyll of 
Photosystem I (chlorophyll b), respectively. In addition, the fluorescence induced by $355 \mathrm{~nm}$ laser exhibited a peak at 440 $\mathrm{nm}$ which is caused by ferulic acidand contained more spectra information than that induced by 460 and $556 \mathrm{~nm}$ laser. From Figure 2, it can be known that the fluorescence spectra excited by the $460 \mathrm{~nm}$ laser exhibited the fluorescence peaks at $685 \mathrm{~nm}$ is more intensive than that $740 \mathrm{~nm}$, and the fluorescence characteristics is contrary when $556 \mathrm{~nm}$ laser serves as excitation light source. The fluorescence spectra excited by 355 $\mathrm{nm}$ laser show inconsistent characteristics of fluorescence peaks for different plant species. These results are similar with the investigations of Apostol et al. (2007) and Agati (1998). Thus, LIF LiDAR can be utilized to distinguish the plant species on the basis of these characteristics of the fluorescence peak.
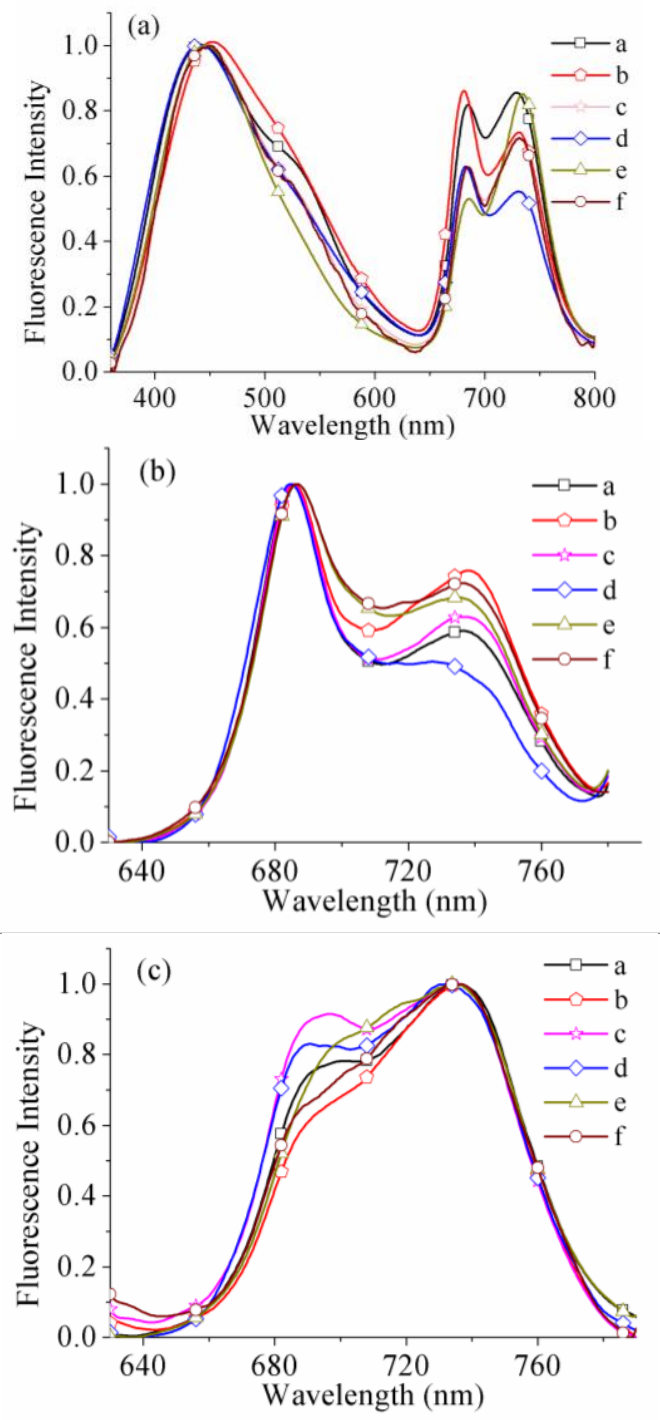

Figure 2. The normalized LIF spectra of the eight plant species were induced by different excitation wavelengths. (a) $C$. yedoensis, (b) C. kotoense, (c) S. babylonica Linn., (d) C. dielsiana, (e) I. chinensis Sims, (f) M. denudata Desr.

The fluorescence spectra excited by different EWs display different fluorescence spectra features (Figure 2). The reason is that fluorescence will be re-absorbed on its path towards the leaf surface. It has been investigated by Agati (1998) and Ramos and Lagorio (2004) in detail. The previous investigation found that carotenoids and chlorophylls in green leaf have a broad absorption band in the $400-500 \mathrm{~nm}$ spectral regions and the re-absorption process by the chlorophyll pigments in the upper layer leaf cells at emission fluorescence spectra between $730-740 \mathrm{~nm}$ is much smaller than that between $680-695 \mathrm{~nm}$. In this study, the $460 \mathrm{~nm}$ excitation light will not penetrate very deeply in the leaf and the fluorescence excited by $460 \mathrm{~nm}$ laser is mainly generated in the mesophyll cells. Therefore, the reabsorption of fluorescence is weak. The fluorescence excited by $556 \mathrm{~nm}$ laser is generated deeper in the leaf tissue than that 460 $\mathrm{nm}$ laser. It will result in a longer pathway and the stronger reabsorption. Hence, these fluorescence spectra excited by 460 and $556 \mathrm{~nm}$ lasers exhibited different spectral shapes. However, it cannot explain the spectra measured by $355 \mathrm{~nm}$ excitation light and still needs to further study in the future.

Then, PCA was used to reconstruct the fluorescent feature vectors and to reduce the dimensionality of the spectra by analysing major attributes. It was found that when the number of PCs exceeded three, the increase of the explained variance with additional PC was reduced to less than $1 \%$. Then, in the three-dimensional coordinate system on the basis of PC1 and PC2, six plant species cannot be distinguished from each other, (Fig. 3).
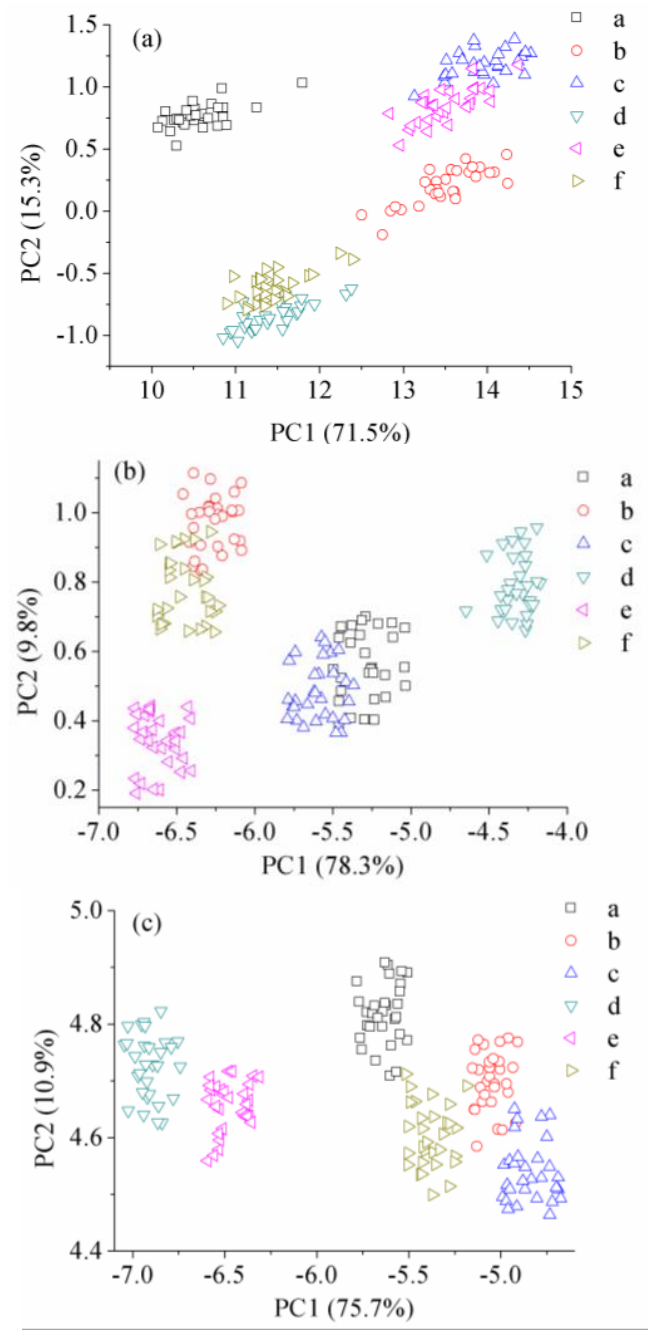

Figure 3. The principle component analysis scores of eight vegetation species under different excitation wavelengths: (a) $355 \mathrm{~nm}$; (b) $460 \mathrm{~nm}$; (c) $556 \mathrm{~nm}$. 
In order to comprehensively analyse the ability of fluorescence spectra excited by different EWs for the identification of plant species, the SVM was then employed to distinguish these plant species on the basis of the variables calculated by PCA. 240 sets of experimental data of each EW were randomly separated into two groups: 180 sets designated as the training set for training SVM and the remaining 60 sets employed as the testing set to verify the tentative performance of the model.

\section{CONCLUSION}

In summary, the fluorescence spectra of eight plant species excited by different EWs (355, 460 and $556 \mathrm{~nm}$ ) were analyzed and found that different EWs will result in different fluorescence spectra shapes. The probable explanation is that the effect of the re-absorption process of the chlorophyll pigments on the fluorescence spectra. The fluorescence peak at $685 \mathrm{~nm}$ is more intensive than that $740 \mathrm{~nm}$ when $460 \mathrm{~nm}$ laser served as excitation light source, and the fluorescence spectra excited by $556 \mathrm{~nm}$ laser display the contrary cases. The fluorescence spectra, which were excited by $355 \mathrm{~nm}$ laser, exhibited inconsistent characteristics of fluorescence peaks for different plant species. The specific reason still needs to further study in the future. Then, PCA combined with SVM was employed to analyze the fluorescence spectra excited by different EWs for identifying plant species. When 355, 460 and $556 \mathrm{~nm}$ lasers served as excitation light sources, the overall identification rates of the eight plant species were $80 \%, 83.3 \%$ and $90 \%$, respectively. Experimental results demonstrated that the $460 \mathrm{~nm}$ EW is superior to $355 \mathrm{~nm}$ EW for the classification of the plant species of the same genus, and is inferior to $556 \mathrm{~nm}$ EW in this study. Therefore, an appropriate EW should be chosen based on practical application requirements and this study can provide investigators with a reference.

\section{REFERENCES}

Agati, G. 1998, Response of the in vivo chlorophyll fluorescence spectrum to environmental factors and laser excitation wavelength. Pure. Appl. Opt. 7, 797-807.

Agarwal N, Gupta S, Pradhan A, Vishwanathan K, Panigrahi PK. 2003, Wavelet transform of breast tissue fluorescence spectra: a technique for diagnosis of tumors. Selected Topics in Quantum Electronics, IEEE J. 9(2):154-161.

Apostol, S.; Viau, A.A.; Tremblay, N. 2007, A comparison of multiwavelength laser-induced fluorescence parameters for the remote sensing of nitrogen stress in field-cultivated corn. Can. J Remote Sens. 33, 150-161.

Chappelle, E.W.; Wood, F.M.; McMurtrey, J.E.; Newcomb, W.W. 1984, Laser-induced fluorescence of green plants. 1: A technique for the remote detection of plant stress and species differentiation. Appl. Opt. 23, 134-138.

Chappelle, E.W.; Wood, F.M.; Wayne Newcomb, W.; McMurtrey, J.E. 1985, Laser-induced fluorescence of green plants. 3: Lif spectral signatures of five major plant types. Appl. Opt. 24, 74-80.

Du, L.; Gong, W.; Shi, S.; Yang, J.; Sun, J.; Zhu, B.; Song, S. 2016, Estimation of rice leaf nitrogen contents based on hyperspectral LIDAR. Int. J. Appl. Earth Obs. Geoinf. 44, 136143.
Günther K, Dahn H-G, Lüdeker W. 1994, Remote sensing vegetation status by laser-induced fluorescence. Remote Sens. Environ. 47(1):10-17.

Gong, W.; Song, S.-L.; Zhu, B.; Shi, S.; Li, F.; Cheng, X. 2012, Multi-wavelength canopy LiDAR for remote sensing of vegetation: Design and system performance. ISPRS J. Photogramm. 69, 1-9.

Koukoulas, S.; Blackburn, G.A. 2005, Mapping individual tree location, height and species in broadleaved deciduous forest using airborne LIDAR and multi-spectral remotely sensed data. Int. J. Remote Sens. 26, 431-455.

Rogers, S.R.; Webster, T.; Livingstone, W.; O’Driscoll, N.J. 2012, Airborne laser-induced fluorescence (LIF) light detection and ranging (LiDAR) for the quantification of dissolved organic matter concentration in natural waters. Estuaries Coasts 35, 959-975.

Ramos, M. E.; Lagorio, M. G. 2004, True fluorescence spectra of leaves," Photochem. Photo. Sci, 3, 1063-1066.

Saito, Y.; Kanoh, M.; Hatake, K.I.; Kawahara, T.D.; Nomura, A. 1998, Investigation of laser-induced fluorescence of several natural leaves for application to lidar vegetation monitoring. Appl. Opt. 37, 431-437.

Yang, J.; Gong, W.; Shi, S.; Du, L.; Sun J.; Song, S.L. 2016, Excitation wavelength analysis of laser-induced fluorescence LiDAR for identifying plant species. IEEE Geosci. Remote S. doi: 10.1109/LGRS.2016.2557484.

Vauhkonen, J.; Hakala, T.; Suomalainen, J.; Kaasalainen, S.; Nevalainen, O.; Vastaranta, M.; Holopainen, M.; Hyyppa, J. 2013, Classification of spruce and pine trees using active hyperspectral LiDAR. IEEE Geosci. Remote S. 10, 1138-1141.

Wu C, Niu Z, Tang Q, Huang W. 2009, Predicting vegetation water content in wheat using normalized difference water indices derived from ground measurements. J. Plant Res. 122(3):317-326. 